\title{
ANÁLISIS MEIÓTICO DE UNA CRUZA ENTRE GIRASOL CULTIVADO (HELIANTHUS ANNUUS L. VAR. MACROCARPUS) Y GIRASOL SILVESTRE (HELIANTHUS ANNUUS L. SSP. TEXANUS HEISER)
}

\author{
Jesús Rodríguez de la Paz, Martha Gómez Martínez y \\ M. Humberto Reyes Valdés \\ Universidad Autónoma Agraria Antonio Narro, Departamento de \\ Fitomejoramiento, 25315 Buenavista, Saltillo, Coahuila, México. \\ mhreyes@uaaan.mx
}

\section{RESUMEN}

En el presente trabajo se compararon los patrones de apareamiento cromosómico meiótico del girasol cultivado (Helianthus annuus L. var. macrocarpus, línea pública HA 89), girasol silvestre (Helianthus annuиs L. ssp. texanus Heiser, procedente de Saltillo, Coahuila) y del híbrido $\mathrm{F}_{1}$. Para ello se analizaron meiocitos en diacinesis y metafase I, basándose en la frecuencia de las configuraciones meióticas. Se evaluó la viabilidad de polen por medio de un método de tinción. El apareamiento cromosómico fue normal en los tres genotipos, con ausencia de univalentes y multivalentes, y sólo se observaron bivalentes en cadena y anillo, por lo que se concluye que los genomas parentales son altamente compatibles en la meiosis. El híbrido presentó un índice de apareamiento cromosómico de 0.82 , próximo al valor medio parental (0.80) y la diferencia del híbrido con cada uno de los taxa parentales (taxon cultivado 0.87 y taxon silvestre 0.75 ) fue altamente significativa, además de variar dentro de las poblaciones estudiadas. Esto indica que el carácter número de quiasmas está bajo control multigénico y se ve afectado por el ambiente. Así pues, una población formada con los progenitores podría ser utilizada para analizar los loci de atributos cuantitativos (QTLs) para apareamiento cromosómico meiótico. El híbrido tuvo un porcentaje de viabilidad de polen (92.58) comparable con el de las plantas parentales (la silvestre 95.57 y la cultivada 95.33). La viabilidad de polen en la $F_{1}$ fue alta, mostró fertilidad, y los genomas de los progenitores fueron altamente compatibles en la meiosis, corroborando que no hay barreras para la reproducción sexual. Esto indica que no hay dificultad en el flujo genético y que se pueden transferir caracteres deseables de H. annuиs silvestre al cultivado.

Palabras clave: análisis meiótico, configuraciones meióticas, Helianthus annuus, hibridación. 


\section{ABSTRACT}

Patterns of meiotic chromosome pairing were compared between three sunflower plants: cultivated sunflower (Helianthus annuus L. var. macrocarpus, public line HA 89), wild sunflower (Helianthus annuus L. ssp. texanus Heiser, collected in Saltillo, Coah.), and the $F_{1}$ hybrid. The study was carried out through analysis of meiocytes in diakinesis and metaphase I, based on the meiotic configuration frequency. Pollen viability was evaluated by means of a staining method. Chromosome pairing was normal in the three materials, with absence of univalents and multivalents, and only chain and ring bivalents were observed, therefore the parental genomes are highly compatible in meiosis. The hybrid showed a chromosome pairing index of 0.82 , close to the midparent value $(0.80)$, and the differences between the hybrid and each parental taxon (cultivated 0.87 , and wild 0.75 ) were highly significant. Besides, this index was variable within populations. This indicates that the chiasma number is under multigenic control and is affected by the environment. Thus, a population formed with both progenitors could be used to analyze the quantitative trait loci (QTLs) for meiotic chromosome pairing. The pollen viability in the hybrid $(92.58 \%)$ was similar to that found in the parental taxa (wild 95.57\%, and cultivated 95.33\%). The pollen viability of the $\mathrm{F}_{1}$ was high, it showed fertility, and the parental genomes were highly compatible in meiosis, confirming that there is no barrier for the sexual reproduction, and that gene flow and transference of desirables characters from wild to cultivated $H$. annuus are easily attainable.

Key words: Helianthus annuus, hybridization, meiotic analysis, meiotic configurations.

\section{INTRODUCCIÓN}

La hibridación amplia ha sido de interés en el girasol, tanto desde el punto de vista teórico como con fines de mejoramiento genético, para transferir genes deseables de las poblaciones silvestres a las líneas cultivadas. Esto es posible, porque se sabe que las plantas espontáneas de girasol están adaptadas a un amplio intervalo de hábitats y poseen considerable variación para la mayoría de las características económicas, agronómicas y de calidad de semilla, para la resistencia a insectos, enfermedades y en general a condiciones ambientales desfavorables (Thompson et al., 1981; Rogers et al., 1982; Seiler, 1988, 1992).

Se han realizado cruzas interespecíficas entre el girasol $H$. annuus y otros representantes de Helianthus, tal es el caso de H. laciniatus (Jackson, 1988) y $H$. tuberosus (Vanozzi, 1994). La hibridación es común entre los girasoles anuales de $H$. secc. Helianthus, y se considera que la integridad de las especies es mantenida 
por fuertes barreras de esterilidad cromosómica, la cual produce semiesterilidad en los híbridos (Rieseberg et al., 1995).

La ocurrencia de irregularidades cromosómicas meióticas en los híbridos $F_{1}$ puede ser debida a que los genomas parentales presentan una homología o compatibilidad reducida, esto es, que los padres difieren en su constitución genómica (Whelan, 1979, 1982; Jackson, 1988; Jan y Chandler, 1989; Atlagic et al., 1993, 1995; Atlagic y Skoric, 1999) o bien a la presencia de uno o pocos genes, tales como los $P h$ (apareamiento homólogo), los cuales son de control del apareamiento cromosómico (Jackson, 1982).

La esterilidad en los híbridos $\mathrm{F}_{1}$ puede ser ocasionada por anormalidades cromosómicas meióticas (Whelan, 1979, 1982; Jackson, 1988; Jan y Chandler, 1989; Atlagic et al., 1993, 1995; Atlagic y Skoric, 1999) y/o a incompatibilidad genética entre los genomas. Se puede hablar de incompatibilidad genética en lugar de incompatibilidad genómica cuando el híbrido tiene comportamiento meiótico regular (Morgan y Thomas, 1991).

Quillet et al. (1995) desarrollaron un mapa genético basado en el cruzamiento de $H$. argophyllus por $H$. annuus. Sin embargo, se observó un alto grado de anomalías cromosómicas en la progenie. Por lo tanto, se debe examinar la compatibilidad genómica de los padres usados en una cruza amplia. Reyes-Valdés y Stelly (1995) han mencionado la utilidad de un análisis meiótico en profase y metafase I para estudios de afinidad genómica entre variedades o especies relacionadas.

Reyes-Valdés et al. (1996) mencionan dos métodos generales para el análisis de quiasmas; uno de ellos consiste en su conteo directo y el otro en la estimación de su frecuencia a través del registro de las configuraciones meióticas en metafase I o diacinesis. Con este último método se estima la frecuencia de la condición quiasmática (esto es, al menos un quiasma) en segmentos cromosómicos específicos, así como el índice de apareamiento cromosómico. Dicho enfoque ha sido usado para estudiar la compatibilidad genómica y las relaciones en híbridos interseccionales (Jackson, 1988), interespecíficos (Jan y Chandler, 1989) e intergenéricos (Thomas y Morgan, 1990; Morgan y Thomas, 1991).

Como parte de la determinación de la compatibilidad genómica del material silvestre con el cultivado es conveniente hacer un estudio meiótico, ya que éste provee información importante sobre las relaciones de apareamiento cromosómico y sobre la posibilidad de transferir caracteres deseables de una especie a otra (Jauhar y Joppa, 1996). Además, con tal información se puede conocer si existe una disminución del entrecruzamiento en la población híbrida, con fines de información básica sobre el comportamiento de la recombinación en cruzamientos entre genotipos distantes. 
El estudio de apareamiento cromosómico en el híbrido puede dar información relevante sobre la genética de la formación de quiasmas. Por lo anterior se fijó como objetivo en el presente trabajo comparar los patrones de apareamiento cromosómico meiótico del girasol cultivado (Helianthus annuus L. var. macrocarpus), del girasol silvestre (Helianthus annuus L. ssp. texanus Heiser) y del híbrido $\mathrm{F}_{1}$.

\section{MATERIALES Y MÉTODOS}

El material de girasol cultivado (Helianthus annuus L. var. macrocarpus, $2 \mathrm{n}$ =34) utilizado como progenitor femenino de la cruza fue la línea pública androestéril CMS HA 89. El silvestre (Helianthus annuus L. ssp. texanus Heiser, 2n = 34), empleado como masculino, estuvo representado por dos plantas (identificadas como $\mathrm{M}_{1} \mathrm{y}_{2}$ ) provenientes de la población ocho (Ac-8) de la colección de girasol silvestre (Helianthus annuus L. ssp. texanus Heiser) del Laboratorio de Análisis de Genomas Vegetales, Departamento de Fitomejoramiento, Universidad Autónoma Agraria Antonio Narro (UAAAN).

El cruzamiento entre el material cultivado y el silvestre se llevó al cabo en el verano de 1999 en el campo experimental de la UAAAN, en Buenavista, Saltillo, Coahuila. Para ello se recolectó polen de plantas silvestres en cajas de Petri, y se espolvorizó un total de tres veces durante la floración en los capítulos del girasol cultivado androestéril CMS HA 89, con la utilización de aplicadores de franela. Para evitar la contaminación con polen extraño, las cabezuelas de ambos progenitores se mantuvieron tapadas con bolsas de papel a lo largo del periodo de la antesis.

En cada uno de los individuos silvestres se efectuaron autopolinizaciones para perpetuar su composición genética y realizar el estudio meiótico. Para ello se taparon los capítulos con bolsas de papel y se frotaron tres veces durante el período de floración con aplicadores de franela, distribuyendo el polen de los mismos sobre los estigmas receptivos. Las plantas provenientes de la autofecundación de $\mathrm{M}_{1} \mathrm{y} \mathrm{M}_{2}$ se designaron como $\mathrm{M}_{1} \otimes \mathrm{y} \mathrm{M}_{2} \otimes$, donde el símbolo $\otimes$ significa autopolinización.

A principios de la primavera del año 2000 se sembraron en vivero los materiales cultivados, silvestres e híbridos en vasos de polipropileno. Posteriormente se trasplantaron a macetas-bolsas de plástico y se distribuyeron bajo el esquema de un diseño completamente al azar con submuestreo. De esta forma, el arreglo experimental quedó estructurado como sigue: tratamientos conformados por las poblaciones evaluadas, repeticiones representadas por plantas individuales dentro de poblaciones, y submuestras compuestas por un número variable, pero mayor que 
100, de meiocitos analizados por planta. La lista de genotipos y tamaños de muestra se anota en el Cuadro 1.

Cuadro 1. Resultados del análisis meiótico efectuado en poblaciones cultivadas, silvestres e híbridas de girasol.

\begin{tabular}{llrccc}
\hline Genotipo & \multicolumn{1}{c}{ Población } & Plantas & $\begin{array}{c}\text { Meiocitos } \\
\text { analizados }\end{array}$ & I & $\begin{array}{c}\text { Frecuencia relativa } \\
\text { de IIc }\end{array}$ \\
\hline Cultivado HA 89 & 15 & 224 & 0.87356 & 0.25289 \\
Silvestre & Ac-8 & 7 & 101 & 0.77402 & 0.45195 \\
Silvestre & $\mathrm{M}_{1} \otimes$ & 8 & 118 & 0.74875 & 0.50249 \\
Silvestre & $\mathrm{M}_{2} \otimes$ & 8 & 120 & 0.73971 & 0.52059 \\
Híbrido & CMS HA 89 x M & 8 & 119 & 0.81241 & 0.37519 \\
Híbrido & CMS HA 89 x M & 7 & 104 & 0.83682 & 0.32636 \\
\hline
\end{tabular}

I = Índice promedio de apareamiento meiótico.; IIc = Bivalentes en cadena; $\mathrm{CMS}=$ Androesterilidad citoplásmica.

Para el estudio citológico de los microsporocitos jóvenes, los botones florales fueron fijados en alcohol de $96^{\circ}$ y ácido acético glacial 3:1 v/v. Para las preparaciones meióticas se utilizó el método de aplastado y tinción de los cromosomas con acetocarmín al 1\%. El polen fue tratado con el colorante citoplásmico Buffalo Black NBR (Jackson, 1988), donde los granos no teñidos se consideraron como abortivos. El número de plantas y el número de determinaciones de viabilidad de polen de cada población se muestran en el Cuadro 2.

Cuadro 2. Resultados del análisis de viabilidad de polen efectuado en poblaciones cultivadas, silvestres e híbridas de girasol.

\begin{tabular}{llccc}
\hline Genotipo & \multicolumn{1}{c}{ Población } & Plantas & Determinaciones & Viabilidad de polen (\%) \\
\hline Cultivado & HA 89 & 3 & 6 & 95.33 \\
Silvestre & Ac- 8 & 7 & 28 & 95.71 \\
Silvestre & $\mathrm{M}_{1} \otimes$ & 7 & 28 & 94.18 \\
Silvestre & $\mathrm{M}_{2} \otimes$ & 8 & 32 & 96.66 \\
Híbrido & CMS HA 89 $\times \mathrm{M}_{1}$ & 6 & 24 & 96.21 \\
Híbrido & CMS HA 89 $\mathrm{x} \mathrm{M}_{2}$ & 7 & 26 & 89.23 \\
\hline
\end{tabular}


Se realizó el análisis en diacinesis y metafase I, registrando el número de bivalentes en cadena (IIc) y en anillo (IIa) formados en cada meiocito, para así determinar los patrones de quiasmas (Jackson, 1988; Reyes-Valdés y Stelly, 1995; Reyes-Valdés et al., 1996). Se utilizó como índice de apareamiento cromosómico la frecuencia de brazos cromosómicos con quiasma (Jackson, 1988), definido como: $\mathrm{I}=[2(\mathrm{IIa})+\mathrm{IIc}] / 34$.

Se emplearon procedimientos de modelos lineales del paquete SAS (Littell et al., 1991) para efectuar el ANOVA (análisis de varianza) bajo el modelo del diseño completamente al azar con submuestreo, la prueba de Tukey de comparación de medias y el análisis de correlación de las variables índice de apareamiento cromosómico y viabilidad de polen. Para el análisis de correlación se contó con 21 plantas del taxon silvestre y 12 del híbrido. Para la variable índice de apareamiento cromosómico también se llevó al cabo un ANOVA bajo el modelo del diseño completamente al azar utilizando sólo la línea HA 89, con el fin de investigar una posible variación de origen ambiental.

Para reforzar o confirmar los resultados tanto del análisis de varianza como de la prueba de comparación de medias de la variable índice de apareamiento cromosómico, se utilizó la Prueba de Neyman-Pearson de la Razón Generalizada de Verosimilitud (GLRT) para bondad de ajuste, llamada también prueba G (Larsen y Marx, 1986; Lynch y Walsh, 1998; Edwards, 1992).

El valor estadístico de prueba con este procedimiento es $G=-2 \ln \lambda$, donde $\lambda$ es la razón generalizada de verosimilitud y se calcula como el cociente de la verosimilitud bajo la hipótesis nula $\left(\mathrm{H}_{\mathrm{o}}\right)$ sobre la verosimilitud bajo la hipótesis alternativa $\left(\mathrm{H}_{\mathrm{a}}\right)$. Una prueba aproximada de la razón generalizada de verosimilitud al nivel de significancia $\alpha$ para $\mathrm{H}_{o}$ contra $\mathrm{H}_{\mathrm{a}}$ se obtiene por rechazar $\mathrm{H}_{\mathrm{o}}$ cuando el valor estadístico de prueba $\mathrm{G}$ es mayor o igual a $\chi_{\alpha,(k-1) \mathrm{g} .1 .}^{2}$, donde en el experimento aquí realizado $\mathrm{k}$ es el número de genotipos.

Para llevar al cabo las pruebas se estimó la frecuencia relativa de IIc en cada uno de los materiales, y su frecuencia relativa global como la proporción conjunta muestral. Con dicha información se efectuaron las pruebas correspondientes de razón de verosimilitud para diferencias entre genotipos en cuanto a frecuencias relativas de cadenas. El valor de $\lambda$ se calculó como el cociente de la verosimilitud de la hipótesis de que las frecuencias de IIc en un conjunto de tratamientos son iguales $\left(\mathrm{H}_{\mathrm{o}}\right)$, sobre la correspondiente a la hipótesis de que existen diferencias entre las mismas $\left(\mathrm{H}_{\mathrm{a}}\right)$.

\section{RESULTADOS Y DISCUSIÓN}

El apareamiento cromosómico meiótico fue normal en $H$. annuus cultivado y silvestre, así como en el híbrido $\mathrm{F}_{1}$. Se observó la ausencia de univalentes y mul- 
tivalentes, y sólo fueron detectados IIa y IIc (Fig. 1). Esto indica que los genomas parentales son altamente homólogos o compatibles en la meiosis.
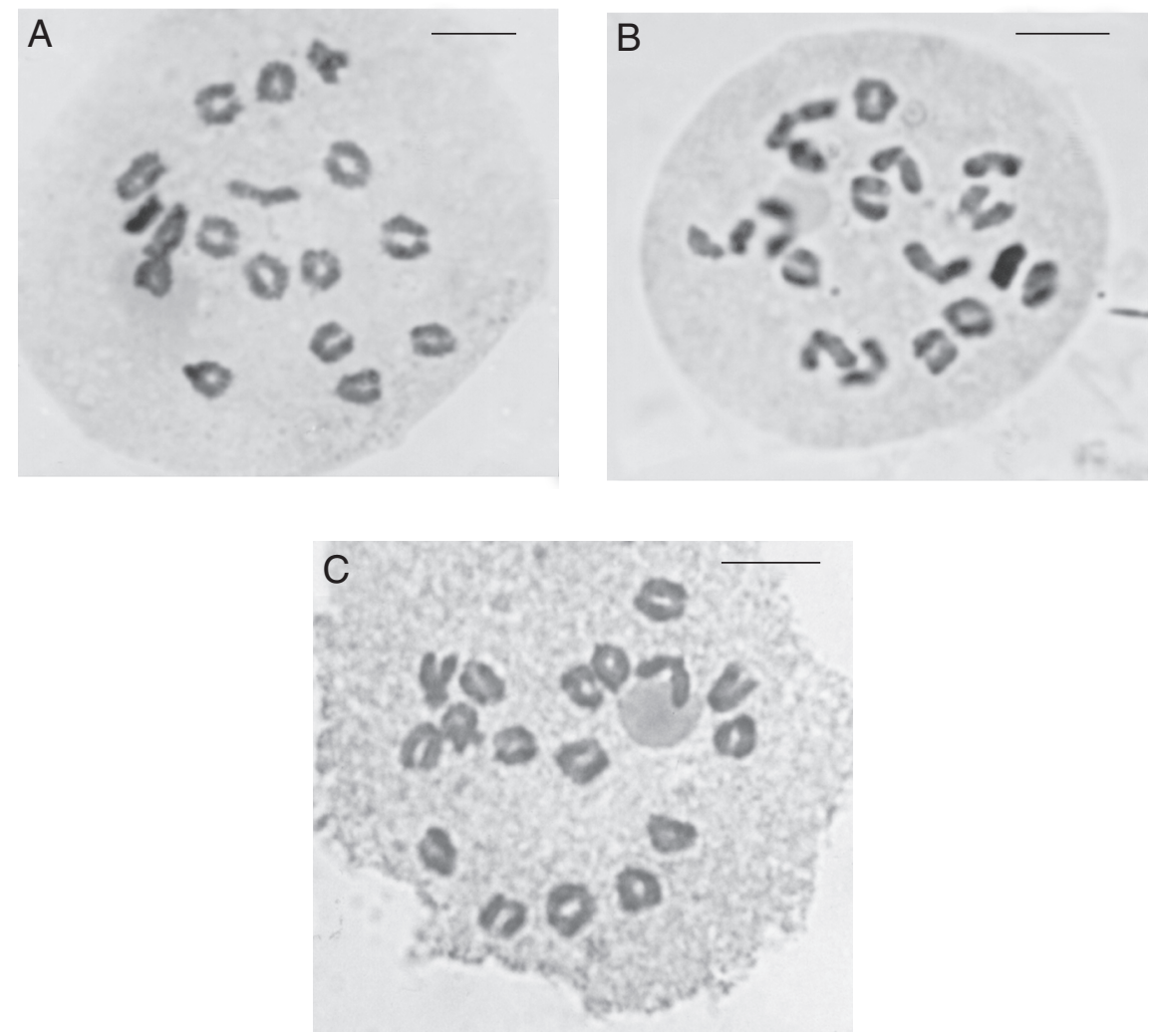

Fig. 1. Configuraciones meióticas en la diacinesis de: (a) girasol cultivado (Helianthus annuus L. var. macrocarpus), línea HA 89, 2n = 34, con 15 IIa y 2IIc; (b) girasol silvestre (Helianthus annuиs L. ssp. texanus Heiser), $2 \mathrm{n}=34$, planta $\mathrm{M}_{1}$, con 9 IIa y 8 IIc; y (c) híbrido $\mathrm{F}_{1}$ entre el material cultivado y el silvestre, CMS HA 89 x M $_{1}, 2 n=34$, con 10 IIa y 7 IIc. La escala en la parte superior derecha de cada fotografía equivale a $10 \mu \mathrm{m}$.

En el Cuadro 1 se presentan las medias del índice de apareamiento cromosómico y de la frecuencia relativa de IIc del material genético utilizado para el análisis meiótico. En ambos casos se observó que las plantas híbridas presentaron un comportamiento consistentemente intermedio entre el de los genotipos cultivados y silvestres. 
Los cuadrados medios del análisis de varianza del índice de apareamiento cromosómico se presentan en el Cuadro 3. Se detectaron diferencias altamente significativas entre los genotipos así como entre plantas dentro de las poblaciones silvestres e híbridas, lo cual parece deberse tanto a la variación genética como a la inducida por el hábitat. Para investigar posibles efectos ambientales sobre el índice de apareamiento cromosómico se realizó un ANOVA con solamente la línea HA 89 (Cuadro 4). Se observaron diferencias altamente significativas entre plantas (de la línea HA 89), lo que muestra que el índice de apareamiento cromosómico presenta un componente de variación de origen no genético.

Cuadro 3. Cuadrados medios del análisis de varianza del índice de apareamiento cromosómico.

\begin{tabular}{lcl}
\hline \multicolumn{1}{c}{ Fuente de variación } & Grados de libertad & Cuadrado medio \\
\hline Genotipos & 2 & $0.99847671^{* *}$ \\
Poblaciones / Genotipos & 3 & 0.03448568 \\
Plantas / Pob. Genotipos & 47 & $0.02458430^{* *}$ \\
Error de muestreo & 733 & 0.00336513 \\
\hline
\end{tabular}

$* *=\mathrm{P} \leq 0.01 ;$ C.V. $(\%)=19.42$

Cuadro 4. Cuadrados medios del análisis de varianza del índice de apareamiento cromosómico en la línea HA 89.

\begin{tabular}{lcc}
\hline Fuente de variación & Grados de libertad & Cuadrado medio \\
\hline Plantas & 14 & $0.01132295^{* *}$ \\
Error experimental & 209 & 0.00260032 \\
\hline
\end{tabular}

$* *=\mathrm{P} \leq 0.01 ;$ C.V. $(\%)=5.84$

Los resultados anteriores indican que la variación en la frecuencia de quiasmas tiene un origen multigénico asociado con efectos ambientales, y concuerdan con la hipótesis de Jackson (1988), quien postula la existencia de varios genes determinantes de la diversidad en número de quiasmas. También coinciden con lo encontrado por Morgan et al. (1986), quienes al analizar el apareamiento cromosómico en una población $\mathrm{F}_{2}$ de Festuca donax x Festuca drymeja, encontraron que la variación en promedio de quiasmas ( $F$. drymeja 8.85 y $F$. donax 11.47) tiene un componente multigénico. 
Las medias de todos los genotipos, comparadas con la prueba de Tukey, resultaron ser significativamente diferentes entre sí (Cuadro 5), con un valor en el híbrido (0.82) intermedio entre los de los progenitores ( 0.87 y 0.75 para el cultivado y silvestre, respectivamente).

Cuadro 5. Prueba de comparación de medias del índice de apareamiento cromosómico. Las medias con letras distintas son estadísticamente diferentes (Tukey, 0.01)

\begin{tabular}{lc}
\hline \multicolumn{1}{c}{ Genotipo } & Media \\
\hline Cultivado (HA 89) & $0.87356 \mathrm{a}$ \\
Híbrido & $0.82379 \mathrm{~b}$ \\
Silvestre (Ac-8) & $0.75308 \mathrm{c}$ \\
\hline
\end{tabular}

A partir de los registros del número de cadenas y anillos se estimó con máxima verosimilitud la frecuencia relativa de IIc para cada material como su proporción muestral, y su error estándar (Cuadro 6). En el Cuadro 7 se presentan las pruebas de Razón Generalizada de Verosimilitud para las frecuencias relativas de IIc de las plantas cultivadas, silvestres e híbridas de girasol. Se detectaron diferencias altamente significativas entre los genotipos, tanto en la prueba global como en la comparación por pares. Esto demuestra contundentemente la existencia de una clara variación en el apareamiento cromosómico entre los tipos de planta analizados.

Cuadro 6. Estimación de la frecuencia relativa de IIc* de cada genotipo.

\begin{tabular}{lcc}
\hline \multicolumn{1}{r}{ Genotipo } & Frecuencia estimada & Error estándar \\
\hline Cultivado (HA 89) & $\theta_{1}=0.25289$ & 0.00704 \\
Híbrido & $\theta_{3}=0.35241$ & 0.00776 \\
Silvestre (Ac-8) & $\theta_{2}=0.49384$ & 0.00659 \\
\hline
\end{tabular}

*IIc $=$ Bivalentes en cadena

Es claro que el híbrido presentó una incidencia de quiasmas manifiestamente distinta de las de sus progenitores y a su vez intermedia entre las de ambos (Cuadro 1). Los promedios de IIc para la línea HA 89, el híbrido y la población silvestre fueron 4.30, 5.99 y 8.40, respectivamente (la media parental fue 6.77). Aquí cabe destacar nuevamente que se observó una gran discrepancia en el apareamiento meiótico 
Cuadro 7. Prueba de Razón Generalizada de Verosimilitud (GLRTs) en la frecuencia relativa de IIc de plantas cultivadas, silvestres e híbridas de girasol. $\mathrm{H}_{\mathrm{o}}=$ Hipótesis nula; $\mathrm{G}=$ estadístico de prueba de razón generalizada de verosimilitud.

\begin{tabular}{lr}
\hline \multicolumn{1}{c}{$\mathrm{H}_{\mathrm{o}}$} & \multicolumn{1}{c}{$\mathrm{G}$} \\
\hline$\theta_{1}=\theta_{3}=\theta_{2}$ & $595.781264^{* *}$ \\
$\theta_{1}=\theta_{3}$ & $89.471550^{* *}$ \\
$\theta_{1}=\theta_{2}$ & $571.808790^{* *}$ \\
$\theta_{3}=\theta_{2}$ & $187.538516^{* *}$ \\
\hline
\end{tabular}

$* *=\mathrm{P} \leq 0.01 ;$ IIc $=$ Bivalentes en cadena; $\theta_{1}, \theta_{2}, \mathrm{y} \theta_{3}=$ frecuencias relativas de IIc de las plantas cultivadas, silvestres e híbridas de girasol;

entre plantas domésticas y no domésticas, ya que en este último la incidencia de IIc fue de casi el doble que en las plantas cultivadas. En el trabajo de Morgan et al. (1986) la diferencia en el apareamiento meiótico entre $F$. donax y $F$. drymeja es también notable, ya que la segunda tuvo una ocurrencia de IIc (5.15) de casi el doble en relación al valor observado en la primera (2.53).

Utilizando las relaciones lineales encontramos que las medias del número de brazos cromosómicos con quiasmas para los materiales cultivado, híbrido y silvestre fueron 29.70, 28.10, y 25.60, respectivamente (la media parental del número de brazos cromosómicos apareados fue 27.23). Esta situación es semejante a lo encontrado por Morgan et al. (1986) en el estudio de un cruzamiento entre Festuca donax x Festuca drymeja, donde estimaron una frecuencia media de quiasmas de 11.47 en $F$. donax, de 8.85 en $F$. drymeja y de 9.65 en el híbrido, esta última cifra es muy cercana al promedio de las dos especies parentales. Tales resultados se asemejan a los encontrados por Jackson (1988) en el análisis de un cruzamiento entre Helianthus laciniatus x Helianthus annuus silvestre, donde la cifra fue de 20.58 en $H$. laciniathus, de 24.76 en $H$. annuus silvestre y de 23.95 en la $\mathrm{F}_{1}$; aunque se detectaron multivalentes y otras irregularidades cromosómicas meióticas en el híbrido. El mencionado autor transformó los multivalentes a bivalentes equivalentes y a partir de ellos determinó la frecuencia media de quiasmas en el híbrido (23.95). Es de notarse cierta similitud en la frecuencia media de quiasmas de $H$. annuus silvestre (24.76) en el análisis de Jackson con la que presenta H. annuus silvestre (25.60) en el trabajo del presente artículo. Para ver esta similitud en términos de número promedio de cadenas bivalentes para $H$. annuus silvestre tenemos en el análisis de Jackson un valor de 9.24, mientras que aquí es de 8.40. 
En el Cuadro 2 se presentan las medias de viabilidad de polen (\%) del material genético utilizado. Se encontró que para los taxa silvestre y el cultivado la viabilidad de polen fue muy similar (95.57\% y 95.33\%, respectivamente). Por otra parte, el híbrido presentó un valor de $92.58 \%$, es decir menor que en las plantas parentales. Sin embargo, esto no es consistente, puesto que mientras una de las poblaciones $\mathrm{F}_{1}$ tuvo un porcentaje de 96.21, muy similar a la de los progenitores, la otra mostró 89.23.

En el Cuadro 8 se muestran los cuadrados medios del análisis de varianza de la viabilidad de polen transformada con la función $\operatorname{ArcSen} \sqrt{ } \mathrm{x} / 100$. Se puede observar que existen diferencias significativas entre los genotipos, y altamente significativas entre poblaciones dentro de los mismos y entre plantas dentro de poblaciones.

Cuadro 8. Análisis de varianza del porcentaje de viabilidad de polen.

\begin{tabular}{lcc}
\hline \multicolumn{1}{c}{ Fuente de variación } & Grados de libertad & Cuadrado medio \\
\hline Genotipos & 2 & $193.343^{*}$ \\
Poblaciones / Genotipos & 3 & $342.358^{* *}$ \\
Plantas / Pob. Genotipos & 32 & $39.368^{* *}$ \\
Error de muestreo & 106 & 6.728 \\
\hline
\end{tabular}

$*=\mathrm{P} \leq 0.51 ; * *=\mathrm{P} \leq 0.01$; Variable transformada con la función Arco Seno.

Las diferencias entre plantas dentro de poblaciones pueden deberse a la variación genética y ambiental existente entre los individuos clasificados dentro del mismo tratamiento. La diversidad entre los genotipos y poblaciones dentro de los mismos se debió sólo al comportamiento divergente del híbrido CMS HA 89 × $\mathrm{M}_{2}$ (Cuadro 2).

La diferencia entre poblaciones $F_{1}$ puede deberse a la variación genética entre las mismas (Atlagic et al., 1993). Otra posible explicación es que la menor viabilidad de polen del cruzamiento CMS HA $89 \times \mathrm{M}_{2}$ obedece a interacciones genéticas, como cierto grado de incompatibilidad entre las plantas de la línea CMS HA 89 y la silvestre $\mathrm{M}_{2}$.

Se puede establecer que, en general, el híbrido presentó un porcentaje de viabilidad de polen (92.58) comparable al de los taxa parentales silvestre y cultivado ( 95.57 y 95.33 , respectivamente). Además, los valores en la $\mathrm{F}_{1}(96.21 \%$ y $89.23 \%$ ) son altos y permiten la reproducción sexual.

El análisis de correlación, en el cual se obtuvo un coeficiente de -0.29 (no significativo), indica que la variación en frecuencia de quiasmas en las poblaciones estudiadas no afectó la viabilidad de polen. 


\section{CONCLUSIONES}

La línea cultivada de girasol HA 89 y la población silvestre de $H$. annuus ssp. texanus presentaron índices de apareamiento iguales a 0.87 y 0.75 , respectivamente, y el híbrido mostró un comportamiento intermedio que correspondió a 0.80 . Además, dicho índice fue variable dentro de las poblaciones. Esto indica la existencia de polimorfismo genético involucrado en la variación de la frecuencia de quiasmas, y que tal característica está bajo control multigénico y es afectada por el ambiente. De aquí se deriva que una estructura familiar formada por estos progenitores podría ser utilizada para analizar loci de caracteres cuantitativos (QTLs) relacionados con el apareamiento cromosómico meiótico.

La meiosis fue normal, con ausencia de univalentes y multivalentes y sólo se observaron IIc y IIa, lo que indica que los genomas parentales son altamente compatibles en la meiosis. El híbrido, que mostró fertilidad, tuvo una viabilidad de polen alta, lo que corrobora que no hay barreras para la reproducción sexual, indicando que existe flujo genético entre las plantas parentales y que se pueden transferir caracteres deseables de $H$. annuиs silvestre al cultivado.

Los resultados obtenidos sugieren la posibilidad de que el proceso de domesticación de $H$. annuus haya traído consigo un incremento en el promedio de quiasmas.

\section{AGRADECIMIENTOS}

El presente trabajo fue financiado por el Consejo Nacional de Ciencia y Tecnología y por la Universidad Autónoma Agraria Antonio Narro.

\section{LITERATURA CITADA}

Atlagic, J., B. Dozet y D. Skoric. 1993. Meiosis and pollen viability in Helianthus tuberosus L. and its hybrids with cultivated sunflower. Pl. Breed. 111: 318-324.

Atlagic, J., B. Dozet y D. Skoric. 1995. Meiosis and pollen grain viability in Helianthus mollis, $H$. salicifolius, $H$. maximiliani and their $\mathrm{F}_{1}$ hybrids with cultivated sunflower. Euphytica 81: 259-263.

Atlagic, J. y D. Skoric. 1999. Cytogenetic study of Helianthus laevigatus and its $\mathrm{F}_{1}$ and $\mathrm{BC}_{1} \mathrm{~F}_{1}$ hybrids with cultivated sunflower, Helianthus annuus. Pl. Breed. 118: 555-559.

Edwards, A. W. F. 1992. Likelihood. John Hopkins University Press. Baltimore. 275 pp. 
Jackson, R. C. 1982. Polyploidy and diploidy: new perspectives on chromosome pairing and its evolutionary implications. Amer. J. Bot. 69: 1512-1523.

Jackson, R. C. 1988. A quantitative cytogenetic analysis of an intersectional hybrid in Helianthus (Compositae). Amer. J. Bot. 75: 609-614.

Jan, C. C. y J. M. Chandler. 1989. Sunflower interspecific hybrids and amphiploids of Helianthus annuus x H. bolanderi. Crop Sci. 29: 643-646.

Jauhar, P. P. y L. R. Joppa. 1996. Chromosome pairing as a tool in genome analysis: merits and limitations. In: Jauhar, P. P. (ed.). Methods of genome analysis in plants. CRC Press. Nueva York. pp. 9-37.

Larsen, R. J. y M. L. Marx. 1986. An introduction to mathematical statistics and its applications. 2a ed. Prentice-Hall. Englewood Cliffs. 630 pp.

Littell, R. C., R. J. Freund y P. C. Spector. 1991. SAS system for linear models. SAS Institute Inc., Cary. 329 pp.

Lynch, M. y B. Walsh. 1998. Genetics and analysis of quantitative traits. Sinauer Associates, Inc. Sunderland. $980 \mathrm{pp}$.

Morgan, W. G. y H. Thomas. 1991. A study of chromosome association and chiasma formation in the amphidiploid between Lolium multiflorum and Festuca drymeja. Heredity 67: 241-245.

Morgan, W. G., H. Thomas, M. Evans y M. Borrill. 1986. Cytogenetic studies of interspecific hybrids between diploid species of Festuca. Can. J. Genet. Cytol. 28: 921-925.

Quillet, M. C., N. Madjidian, V. Griveau, H. Serieys, M. Tersac, M. Lorieux y A. Berville. 1995. Mapping genetic factors controlling pollen viability in an interspecific cross in Helianthus sect. Helianthus. Theor. Appl. Genet. 91: 1195-1202.

Reyes-Valdés, M. H. y D. M. Stelly. 1995. A maximum likelihood algorithm for genome mapping of cytogenetic loci from meiotic configuration data. Proc. Natl. Acad. Sci. U.S.A. 92: 9824-9828.

Reyes-Valdés, M. H., Y. Ji, C. F. Crane, J. F. Taylor, M. N. Islam-Faridi, H. J. Price y D. M. Stelly. 1996. ISH-facilitated analysis of meiotic bivalent pairing. Genome 39: 784792.

Rieseberg, L. H., A. M. Desrochers y S. J. Youn. 1995. Interspecific pollen competition as a reproductive barrier between sympatric species of Helianthus (Asteraceae). Amer. J. Bot. 82: 515-519.

Rogers, C. E., T. E. Thompson y G. J. Seiler. 1982. Sunflower species of the United States. Natl. Sunflower Assoc. Bismarck. 75 pp.

Seiler, G. J. 1988. The genus Helianthus as a source of genetic variability for cultivated sunflower. In: Proc.12th Int. Sunflower Conf., Novi Sad, Yugoslavia. 25-29 July 1988. Int. Sunflower Assoc. Paris, France. Vol. 1 pp. 17-58.

Seiler, G. J. 1992. Utilization of wild sunflower species for the improvement of cultivated sunflower. Field Crops Res. 30: 195-230.

Thomas, H. M. y W. G. Morgan. 1990. Analysis of synaptonemal complexes and chromosome pairing at metaphase I in the diploid intergeneric hybrid Lolium multiflorum x Festuca drymeja. Genome 33: 465-471.

Thompson, T. E., D. C. Zimmerman y C. E. Rogers. 1981. Wild Helianthus as a genetic resource. Field Crops Res. 4: 333-343. 
Vanozzi, G. P. 1994. Study of embryogenesis in interspecific hybrids. In: Seiler, G. J. (ed.). FAO working group: evaluation of wild Helianthus species. Progress report 19911993. pp. 117-118.

Whelan, E. D. P. 1979. Interspecific hybrids between Helianthus petiolaris Nutt. and $H$. annuus L.: effect of backcrossing on meiosis. Euphytica 28: 297-308.

Whelan, E. D. P. 1982. Trisomic progeny from interspecific hybrids between Helianthus maximiliani and H. annuus. Can. J. Genet. Cytol. 24: 375-384.

Recibido en febrero de 2005.

Aceptado en abril de 2007. 